\title{
Correction: Genomic Analysis of a Mycobacterium Bovis Bacillus Calmette- Guérin Strain Isolated from an Adult Patient with Pulmonary Tuberculosis
}

The PLOS ONE Staff

\section{Notice of Republication}

This article was republished on May 14, 2015, to correct an error in the title: "Bovis Bacillus" was incorrectly displayed as one word. Please download this article again to view the correct version. The originally published, uncorrected article and the republished, corrected article are provided here for reference.

\section{Supporting Information}

S1 File. Originally published, uncorrected article.

(PDF)

S2 File. Republished, corrected article.

(PDF)

\section{Reference}

1. Li X, Chen L, Zhu Y, Yu X, Cao J, Wang R, et al. (2015) Genomic Analysis of a Mycobacterium Bovis Bacillus Calmette-Guérin Strain Isolated from an Adult Patient with Pulmonary Tuberculosis. PLoS ONE 10(4): e0122403. doi: 10.1371/journal.pone.0122403 PMID: 25876043
G openaccess

Citation: The PLOS ONE Staff (2015) Correction: Genomic Analysis of a Mycobacterium Bovis Bacillus Calmette-Guérin Strain Isolated from an Adult Patient with Pulmonary Tuberculosis. PLoS ONE 10(5): e0129753. doi:10.1371/journal.pone. 0129753

Published: May 29, 2015

Copyright: $\odot 2015$ The PLOS ONE Staff. This is an open access article distributed under the terms of the Creative Commons Attribution License, which permits unrestricted use, distribution, and reproduction in any medium, provided the original author and source are credited. 